\title{
USO DEL ESPACIO DOMÉSTICO. UN ANÁLISIS COMPARATIVO DE SUS ESCENARIOS DE ACTIVIDAD
}

\author{
Eric Orlando Jiménez-Rosas y Óscar Iván Negrete-Rodríguez \\ Facultad de Arquitectura, FES Iztacala, UNAM \\ MÉXICO
}

\begin{abstract}
RESUMEN
Las características ambientales y sociales de las viviendas urbanas y rurales son diferentes, por lo que se espera que los ocupantes usen el espacio de forma distinta. Con la finalidad de conocer las diferencias en el uso del espacio entre las casas de estos dos ambientes, se realizó un estudio sobre el uso del tiempo. Se obtuvieron los patrones de actividad para cada ambiente, resaltándose los aspectos comunes y distintos entre ellos. Durante dos días, uno entre semana y otro en fin de semana, 60 moradores urbanos y 60 rurales, registraron en un diario las actividades que realizaron dentro de sus casas. Se obtuvo información acerca de la frecuencia y duración de las actividades, los días y lugares donde éstas ocurrieron, así como de las personas que estuvieron presentes. Esta información se integró en un análisis contextual (Harvey, 2002), cuyos resultados mostraron que entre las casas urbanas y rurales existen diferentes escenarios dominantes para las actividades domésticas. Estas diferencias se discuten en términos de las estructuras y oportunidades que ofrecen las viviendas para la actividad de sus habitantes, así como la importancia de incorporar a la psicología ambiental, la investigación sobre los asentamientos rurales.
\end{abstract}

Palabras Clave:

Vivienda, Urbano, Rural, Uso del tiempo, Análisis contextual

\section{USE OF DOMESTIC SPACE. URBAN / RURAL COMPARATIVE ANALYSIS OF THEIR ACTIVITY SCENARIOS}

\begin{abstract}
The environmental and social characteristics of urban and rural households are different, so it is expected that the occupants use the space differently. In order to know the differences in the use of space between the houses of these two environments, a time use study was conducted. The activity patterns in the home were obtained for each area, highlighting the common and different features between them. For two days, on weekday and other on weekend, 60 urban and 60 rural inhabitants, reported in a diary the activities they carried out within their homes. The information obtained was about the frequency and duration of the activities, the days and places where those took place and the people who were present. This information was integrated into a contextual analysis (Harvey, 2002), and the results showed that there are different main scenarios for the domestic activity between urban and rural houses. These differences are discussed in terms of the structures and opportunities that the houses offer to the inhabitants, as well as the importance of including within de environmental psychology, the research of the rural settlements.
\end{abstract}

Keywords:

Housing, Urban, Rural, Time use, Contextual analysis, Activity settings

Bitácora del ARtículo:

| Recibido: 1 de Agosto de 2015 | Aceptado: 1 Septiembre de 2015 | Publicado en línea: Enero-Junio de 2016 | 


\title{
USO DEL ESPACIO DOMÉSTICO. ANÁLISIS COMPARATIVO URBANO/RURAL DE SUS ESCENARIOS DE ACTIVIDAD
}

\author{
Eric Orlando Jiménez-Rosas y Óscar Iván Negrete-Rodríguez \\ MÉXICO
}

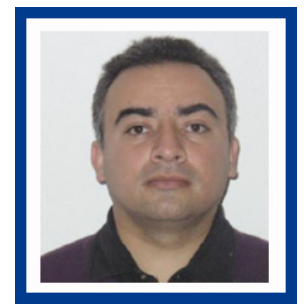

Eric Orlando Jiménez Rosas

Facultad de Arquitectura, UAAP

Correo: orlando.jimenez@ired.unam. $\underline{\mathrm{mx}}$

Dr. en Psicología por la Universidad Nacional Autónoma de México. Profesor en la Unidad Académica de Arquitectura de Paisaje, en la Facultad de Arquitectura de la UNAM.

Ver más...

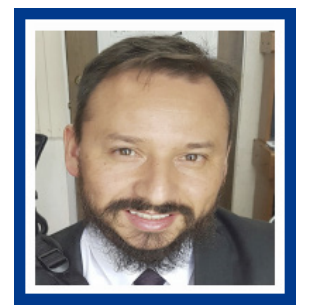

Óscar Iván Negrete Rodríguez FES Iztacala, UNAM

Correo: ivanpunk@gmail.com

Maestro en Psicología por la UNAM, con Residencia en Psicología Organizacional. Profesor de la licenciatura en Psicología y de la maestría en Psicología Organizacional de la FES Iztacala, UNAM.

Ver más...

\section{CONTRIBUCIÓN DE LOS AUTORES}

El autor, Eric Jiménez fue el responsable de la concepción y realización del proyecto, incluyendo la revisión de la literatura, el levantamiento de datos y el análisis de los mismos. I El autor Óscar Negrete contribuyó en el levantamiento de datos y en la realización de los análisis estadísticos pertinentes.

\section{AgRADECIMIENTOS}

Los autores agradecen el apoyo brindado por la Dra. Gemma Verduzco Chirino, de la Facultad de Arquitectura UNAM, y de la Dra. Concepción Sánchez Quintanar, del Colegio de Posgraduados.

\section{Datos de Filiación de los Autores}

\begin{abstract}
Unidad Académica de Arquitectura de Paisaje, en la Facultad de Arquitectura, UNAM| Facultad de Estudios Superiores Iztacala, UNAM.
\end{abstract}

\section{Copyright: (C) 2016 Jiménez-Rosas, E. O. \& Negrete-Rodríguez Ó. I.}

Este es un artículo de acceso abierto distribuido bajo los términos de la licencia Creative Commons Reconocimiento-NoComercial -Compartirlgual 4.0 Internacional, por lo que su contenido gráfico y escrito se puede compartir, copiar y redistribuir total o parcialmente sin necesidad de permiso expreso de sus autores con la única condición de que no se puede usar con fines directamente comerciales y los términos legales de cualquier trabajo derivado deben ser los mismos que se expresan en la presente declaración. La única condición es que se cite la fuente con referencia a la Revista Digital Internacional de Psicología y Ciencia Social y a sus autoras. 


\section{TABLA DE CONTENIDO}

INTRODUCCIÓN

40

Patrones de actividad doméstica, 40

MÉTOdo

Participantes, 41

Materiales y Procedimiento, 41

Mediciones, 42

Análisis estadísticos, 42

REsultados

Frecuencias de las actividades, 42

Contacto social, 43

Duración de la actividad, 43

Escenarios de actividad: condiciones dominantes de la actividad

doméstica, 43

Hipercódigos del trabajo en casa, 44

Hipercódigos del esparcimiento, cultura y entretenimiento, 44

Hipercódigos de los cuidados personales, 44

Discusión

CONCLUSIONES 


\section{INTRODUCCIÓN}

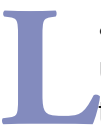

a psicología se ha caracterizado por un marcado urbanocentrismo, es decir, una tendencia a prestar más atención hacia los problemas urbanos que a los rurales. Esta tendencia también está presente en otras disciplinas científicas y en el común de la población. De acuerdo a Baptista (2001), el urbanocentrismo puede estar asociado al portentoso crecimiento de las ciudades durante el último siglo y al lugar de residencia de los científicos. Actualmente la mitad de la población del mundo vive en las ciudades y la otra en zonas rurales. La Organización de las Naciones Unidas calcula que para el año 2050, el $70 \%$ de la población mundial vivirá en ambientes urbanos, siendo América Latina la que encabeza esta propensión (United Nations, 2015). Por otro lado, la mayor parte de los científicos residen en ciudades y ahí ubican sus objetos de estudio, quedando así el ambiente rural fuera de su atención.

Se ha reportado que en las zonas rurales la vulnerabilidad es mayor ya que existen grandes disparidades socioeconómicas y acentuadas necesidades de atención a la salud (Helbok, 2003; Hicks, 2002). A pesar de esto, los asentamientos rurales son foco de interés sólo cuando ocurren afectaciones o desastres graves. Las consecuencias de este desinterés no son triviales. En principio, la desatención promueve y mantiene una estigmatización acerca del ambiente rural y sus habitantes. Existe una idea generaliza acerca de que los habitantes rurales son fuertes e independientes, que no requieren ayuda y que viven con tranquilidad y sin estrés. Este estigma fomenta que se subestimen las necesidades apremiantes y reales de los asentamientos rurales.

El estudio de la vivienda en psicología ambiental también presenta este sesgo. Las investigaciones se enfocan marcadamente hacia la vivienda urbana. El modelo de Habitabilidad de la Vivienda Urbana (Mercado, Ortega, Luna \& Estrada, 1995) fue la primera aproximación psicológica al tema en México. En éste se muestra cómo factores compuestos por variables emocionales, operativas y simbólicas, influyen sobre la percepción de habitabilidad. A partir de este modelo se han generado diversas publicaciones y tesis orientadas principalmente hacia la vivienda urbana. Concepción Sánchez, investigadora que ha abordado los aspectos psicológicos de la vivienda rural, comenta que al no encontrarse antecedentes psicológicos sobre el estudio de la vivienda rural, la investigación sobre este tópico tiene que apoyarse en modelos sobre la vivienda urbana (Sánchez, 2009). De esta manera, la comprensión de las transacciones entre los ambientes residenciales y sus moradores será limitada si no se presta mayor interés y atención hacia los asentamientos rurales.

En el campo de la arquitectura se presenta la misma situación. Aguilar (2001) considera que la arquitectura privilegia el estudio de la vivienda urbana sobre la rural. En su opinión, la arquitectura concibe a la vivienda rural como carente de interés y presta más atención hacia la monumentalidad de la vivienda urbana.

Un mismo tipo de ambiente, como la vivienda, puede afectar de forma diferente a las personas dependiendo de sus características, por ejemplo, las asociadas a los asentamientos urbanos y rurales. De manera general, Rapoport (2005) menciona que los distintos ambientes inciden en la fisiología de las personas, como puede ser en la adaptación y acomodación a las condiciones de temperatura, humedad, niveles de luz, ruido, etc.; en el confort anatómico, en la recepción sensorial con respecto al entorno y en el aprendizaje y significado sobre el espacio. Los estudios en psicología ambiental han mostrado algunas de estas influencias. Por ejemplo, el hacinamiento o la vegetación en la vivienda pueden tener diferentes significados y efectos entre los moradores urbanos y rurales (Evans, Saegert \& Harris, 2001; Wells \& Evans, 2003). En relación con las actividades dentro de las casas, Rapoport (2005) menciona que la gran variabilidad en las viviendas tiene un efecto sobre cinco dimensiones: las actividades en sí mismas, cómo se realizan éstas, cómo se asocian entre ellas para formar sistemas de actividades, así como el significado que se les otorga. Por tanto, es necesario prestar atención a las características particulares de cada grupo de residentes acerca de las formas de relacionarse con sus respectivos ambientes.

\section{Patrones de actividad doméstica}

La vida cotidiana en la casa es una experiencia compleja, compuesta por diversos episodios interrelacionados. Los ocupantes de las casas Ilevan a cabo sus rutinas diarias frecuentemente sin la conciencia de esta complejidad. En la vivienda diariamente coexisten, temporal y espacialmente, numerosas actividades. Para cada una de ellas son necesarios diferentes requerimientos. Estas actividades son realizadas por diferentes individuos, con diferentes motivaciones y valoraciones acerca del espacio y la actividad. Además, todas estas condiciones varían dependiendo del contexto físico, social y temporal en las cuales se encuentren (Werner, Altman, \& Oxley, 1985). 
Las actividades de las personas, y las condiciones y ambientes donde éstas se desarrollan, pueden estudiarse mediante procedimientos de observación (Martínez, 1991) o el análisis de escenarios conductuales (Wicker, 1979). Aunque con estos métodos es posible obtener una cantidad indiscutible de actividades, la laboriosidad que implica puede impedir el registro de eventos importantes, además de que el mismo proceso de observación tiende a inhibir la ocurrencia común de actividades (Larson, 1989). Por otro lado, mediante estos métodos no es posible obtener información acerca de aspectos subjetivos (por ejemplo, motivaciones, satisfacción con los espacios o con las actividades) (Michelson, 1994).

En este estudio se utilizó la metodología del uso del tiempo (Michelson, 1990, 1994; Michelson \& Reed, 1975; Pentland, Harvey, Lawton \& McColl, 2002; Robinson, 1988), ya que puede proporcionar patrones de actividad dentro de la vivienda, los cuales se pueden asociar a distintos grupos y condiciones. La metodología de uso del tiempo asume que la rutina diaria de las personas puede ser fragmentada en una serie de categorías conductuales, por ejemplo, comer, dormir o bañarse. A estas actividades se les puede asociar una serie de dimensiones, las cuales, conjuntamente, dan cuenta de su contexto. Estas dimensiones son temporales (cuándo, cuánto tiempo), sociales (con quién), ambientales (dónde) y subjetivas (satisfacción, estrés, presión del tiempo). Estas categorías conductuales (o actividades) y sus dimensiones asociadas, pueden definirse y funcionar de forma independiente; sin embargo, el interés primordial es el revelamiento de patrones globales de conducta a través de la integración de las dimensiones que los componen. Esta metodología es relevante socialmente. Por ejemplo, Pentland y MCcoll (2002) han descrito las diversas formas en las que la investigación sobre el uso del tiempo se vincula con el bienestar. Los resultados de investigaciones sobre este tópico han mostrado que el involucramiento con actividades diversas correlaciona positivamente con la salud y el bienestar.

Este estudio forma parte del proyecto Uso y organización del espacio doméstico. Estudio comparativo entre casas urbanas y rurales, el cual tuvo como objetivo comparar las diferencias en la distribución de la actividad doméstica a través de la configuración del espacio, entre las casas urbanas y rurales (Jiménez \& Verduzco, 2009). El tema principal de dicha publicación fue la sintaxis espacial de las casas urbanas y rurales, medidas a través de su profundidad, y su relación con la ubicación de las actividades. El presente estudio se enfocó en identificar cuáles son los contextos predominantes de las actividades en las casas urbanas y rurales.
El contexto brinda información importante ya que incluye aquellos aspectos ambientales y sociales que son relevantes para la actividad. El contexto de las actividades domésticas incluye dimensiones como la duración de las actividades en la casa, los días y lugares donde éstas ocurren y las personas que estuvieron presentes. La metodología del uso del tiempo ofrece un procedimiento analítico para estudiar el contexto de la actividad, el análisis contextual (Harvey, 2002). Este análisis permite conocer cuál es el contexto más frecuente de las distintas actividades que ocurren en la casa. Al estudiar este contexto, es posible identificar qué subyace a las diferencias en el uso del espacio entre las casas urbanas y rurales. El objetivo de este estudio fue conocer las diferencias que existen en los escenarios de actividad entre las casas urbanas y rurales.

\section{MÉTodo}

\section{Participantes}

El estudio se realizó con 120 participantes, todos ellos mayores de 18 años. El 50\% fueron residentes de la ciudad de México, los cuales vivían en casas habitación unifamiliares de hasta tres pisos. La media de edad de estos participantes fue de 26 años (DE $=5)$. En promedio, estos participantes llevaban viviendo en sus casas 13 años (DE $=7.38$, mínimo $=1$, máximo $=35$ ) y en ellas habitaban, en promedio, cuatro personas $(\mathrm{DE}=1.58)$. Los participantes urbanos fueron 40 mujeres y 20 hombres.

El restante $50 \%$ estuvo conformado por 49 mujeres y 11 hombres, con una edad promedio de 38 años (DE = 11.5), todos ellos residentes de dos localidades: Santa Catarina del Monte y Santa María Tecuanulco, ubicadas en el Municipio de Texcoco, Estado de México. Las viviendas de estos participantes variaban entre cuartos pequeños y casas autoconstruidas de un solo piso. El número de habitantes rurales promedio por casa fue de cinco $(\mathrm{DE}=$ 2.03) y ellos llevaban habitando su casa, en promedio, 17 años ( $\mathrm{DE}=16$, mínimo = 1, máximo 76).

\section{Materiales y Procedimiento}

Se solicitó a los participantes registrar en un diario las actividades que realizaron en casa. El registro se realizó durante las 24 horas de dos días, uno entre semana y otro en fin de semana, comenzando a las 00:00 a.m. y finalizando a las 11:59 pm. El diario estuvo integrado por varias hojas con una matriz en cada una de ellas. La matriz del diario incluyó, en las columnas, las siguientes dimensiones contextuales: el inicio y término de la actividad, número de personas presentes, el lugar de la casa en que es- 
taban ubicados. Cada renglón de la matriz correspondía a una actividad, a la cual se le incorporaba la información de las dimensiones contextuales antes descritas. Los datos de cada renglón conformaban un episodio de actividad. En la Figura 1 se muestra un ejemplo.

\begin{tabular}{lll}
$\begin{array}{r}\text { Hora } \\
\text { INICIO }\end{array}$ & ACtIVIDAD & $\begin{array}{c}\text { HorA } \\
\text { TÉRMINO }\end{array}$ \\
\hline $2: 15$ & Comer & $2: 35$ \\
\hline
\end{tabular}

Figura 1.

Ejemplo del registro de un episodio de actividad

\section{Mediciones}

A partir de los 120 diarios, se obtuvieron 2672 episodios de actividades, de los cuales 1609 corresponden a registros urbanos y 1063 a registros rurales. Las actividades registradas se organizaron en tres categorías representativas de la vida doméstica con base en la Encuesta Nacional sobre el Uso del Tiempo del Instituto Nacional de Geografía, Estadística e Informática (INEGI, 2005):

- Trabajo en casa. Por ejemplo, actividades escolares, preparación de alimentos, limpieza de la vivienda, limpieza y cuidado de la ropa, etcétera.

- Esparcimiento, cultura y entretenimiento. Por ejemplo, ver televisión, escuchar radio o música, pasatiempos, etcétera.

- Cuidados personales. Por ejemplo, alimentación, higiene y arreglo personal, vestimenta, etcétera.

\section{Análisis estadísticos}

Se realizaron análisis descriptivos para conocer las frecuencias y porcentajes de cada categoría de actividad (trabajo en casa, esparcimiento y cuidados personales), así como su distribución por:

- Asentamiento (rural/ urbano)

na)

- Día de la semana (entre semana / fin de sema-

- Contacto social (solo / acompañado)

Se realizaron análisis mediante la prueba t de igualdad de medias para muestras independientes, para cono- cer si existen diferencias en la duración de las actividades entre los asentamientos urbanos y rurales.

También se realizó un análisis contextual para conocer los escenarios de actividad más frecuentes en las casas.

Las dimensiones contextuales de las actividades se dividieron en categorías y a cada una se le asignó un código. Las categorías y códigos asignados se presentan a continuación:

- Duración (DUR) - $\mathrm{C}=$ corta (1 a 14 minutos), $\mathrm{M}=$ media (15 a 29 minutos), $\mathrm{L}=$ larga (más de 15 minutos)

- Ubicación espacial (UE) - $\mathrm{R}=$ recámara, $\mathrm{B}=$ baño, $\mathrm{S}=$ sala, $\mathrm{CM}=$ comedor, $\mathrm{CC}=$ cocina

- Día de la semana (DÍA) - ES= entre semana, FS = fin de semana

- Contacto social (CS) - S = solo, A = acompañado

Posteriormente se identificaron, para cada dimensión contextual, los códigos más frecuentes. Por ejemplo, para la actividad de Trabajo en casa se identificó la duración $(\mathrm{C}=$ corta, $\mathrm{M}=$ media o $\mathrm{L}=$ larga) que se presentó con mayor frecuencia. Este procedimiento se realizó para cada grupo de actividad identificando el código más frecuente de cada dimensión contextual.

Una vez identificados los códigos más frecuentes, estos se agruparon en un hipercódigo, el cual integró a los códigos más frecuentes de cada dimensión contextual. Estos hipercódigos representan los escenarios de actividad, es decir, las condiciones más frecuentes o dominantes de las diferentes actividades.

\section{Resultados}

\section{Frecuencias de las actividades}

Se obtuvieron, en total, 2672 reportes de actividad (Tabla 1). La cantidad de reportes en los días entre semana fue muy semejante a la de los días en fin de semana (50.9\% y $49.1 \%$ respectivamente). Las actividades que más se presentaron fueron las de cuidados personales, seguidas de las del trabajo en casa y de esparcimiento (45.2\%, 34.5\% y $20.3 \%$ respectivamente).

Considerando las tres categorías de actividad, los participantes urbanos reportaron más episodios de actividad que los rurales, tanto en fin de semana como entre semana. El $60.2 \%$ del total de episodios fueron reportados por los habitantes urbanos, mientras que el 39.8\% por los rurales (Tabla 1). 
En las actividades de cuidados personales y de esparcimiento, los habitantes urbanos reportaron más actividades que los rurales, tanto entre semana como en fin de semana. En la actividad de trabajo en casa los residentes rurales reportaron más actividades.

Tabla 1.

Frecuencia de las actividades según el día en la semana

\begin{tabular}{|c|c|c|c|c|c|c|c|}
\hline \multicolumn{8}{|c|}{ Día EN LA SEMANA } \\
\hline & \multicolumn{3}{|c|}{ ENTRE SEMANA } & \multicolumn{3}{|c|}{ FIN DE SEMANA } & \multirow{2}{*}{ TOTAL } \\
\hline & Rural & Urbano & Total & RuRAL & $U_{\text {rbano }}$ & Total & \\
\hline $\begin{array}{l}\text { Trabajo } \\
\text { en casa }\end{array}$ & 303 & 214 & 517 & 182 & 223 & 405 & $\begin{array}{c}922 \\
(34.5)\end{array}$ \\
\hline $\begin{array}{l}\text { Esparcimiento, } \\
\text { cultura y } \\
\text { entretenimiento }\end{array}$ & 85 & 149 & 234 & 70 & 239 & 309 & $\begin{array}{c}543 \\
(20.3)\end{array}$ \\
\hline $\begin{array}{l}\text { Cuidados } \\
\text { personales }\end{array}$ & 211 & 399 & 610 & 212 & 385 & 597 & $\begin{array}{l}1207 \\
(45.2)\end{array}$ \\
\hline Total & $\begin{array}{c}599 \\
(22.4)\end{array}$ & $\begin{array}{c}762 \\
(28.5)\end{array}$ & $\begin{array}{c}1361 \\
(50.9)\end{array}$ & $\begin{array}{c}464 \\
(17.4)\end{array}$ & $\begin{array}{c}847 \\
(31.7)\end{array}$ & $\begin{array}{c}1311 \\
(49.1)\end{array}$ & $\begin{array}{l}2672 \\
(100)\end{array}$ \\
\hline
\end{tabular}

Nota. Los números entre paréntesis representan el porcentaje de actividades.

\section{Contacto social}

Del total de actividades reportadas, el $57.7 \%$ ocurrieron sin compañía, mientras que el $42.3 \%$ ocurrieron en presencia de otras personas (Tabla 2). Esta mayoría se debe a que las actividades más frecuentes en los reportes fueron las de cuidados personales, y éstas se realizaron con mayor frecuencia en ausencia de otras personas. Las actividades de esparcimiento y de trabajo en casa ocurrieron, en mayor medida, en compañía de otros, pero la frecuencia de estas actividades es menor respecto a las de cuidados personales. Las tres categorías de actividad se presentaron en ausencia de otras personas con mayor frecuencia en los reportes urbanos.

Tabla 2.

Frecuencia de las actividades según el contacto social

\begin{tabular}{|c|c|c|c|c|c|c|c|}
\hline \multicolumn{8}{|c|}{ CONTACTO SOCIAL } \\
\hline & \multicolumn{3}{|c|}{ SOLO } & \multicolumn{3}{|c|}{ ACOMPAÑADO } & \multirow{2}{*}{ TOTAL } \\
\hline & RuRAL & Urbano & Total & Rural & URBANo & TotAL & \\
\hline $\begin{array}{l}\text { Trabajo } \\
\text { en casa }\end{array}$ & 254 & 318 & 572 & 231 & 119 & 350 & $\begin{array}{c}922 \\
(34.5)\end{array}$ \\
\hline $\begin{array}{l}\text { Esparcimiento, } \\
\text { cultura y y } \\
\text { entretenimiento }\end{array}$ & 23 & 193 & 216 & 132 & 195 & 327 & $\begin{array}{c}543 \\
(20.3)\end{array}$ \\
\hline
\end{tabular}

Nota. Los números entre paréntesis representan el porcentaje de actividades.

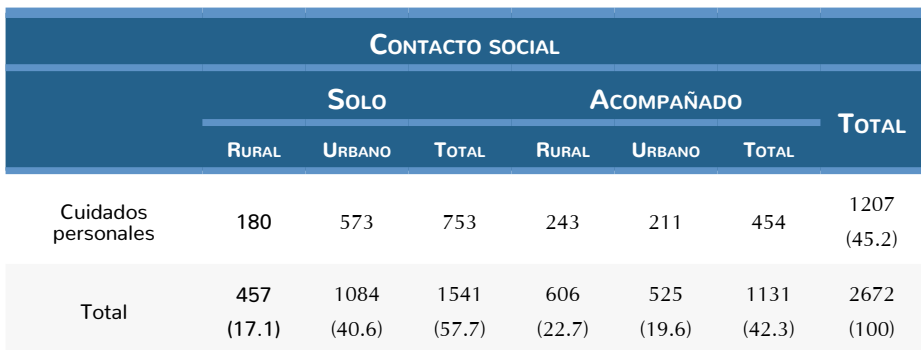

Nota. Los números entre paréntesis representan el porcentaje de actividades.

\section{Duración de la actividad}

Las actividades que tuvieron mayor duración, en promedio, fueron las de trabajo en casa, seguidas, respectivamente, de las de esparcimiento y de cuidados personales (Tabla 3).

Las actividades de trabajo en casa en los días entre semana tienen una duración significativamente mayor en las casas rurales. Los moradores rurales dedicaron los días entre semana, una hora y catorce minutos más que los urbanos al trabajo en casa. En cambio, los habitantes urbanos dedicaron 33 minutos más que los rurales a las actividades de cuidado personal entre semana. Los residentes urbanos también superaron a los rurales por una hora más en las actividades de esparcimiento entre semana (Tabla 3).

Resumiendo, los datos indican que entre semana los residentes urbanos dedicaron más tiempo a sí mismos, en tanto que los habitantes rurales otorgan mayor tiempo al cuidado de la casa y de otras personas.

\section{Tabla 3.}

Uso del tiempo por lugar de residencia y día en la semana (promedio de horas y minutos por día)

\begin{tabular}{|c|c|c|c|c|c|c|}
\hline \multicolumn{7}{|c|}{ DíA EN LA SEMANA } \\
\hline & \multicolumn{3}{|c|}{ ENTRE SEMANA } & \multicolumn{3}{|c|}{ FIN DE SEMANA } \\
\hline & Rural & Urbano & DifERENCIA & Rural & Urbano & DifERENCIA \\
\hline $\begin{array}{l}\text { Trabajo } \\
\text { en casa }\end{array}$ & $3: 47$ & $2: 33$ & $+1: 14^{*}$ & $2: 37$ & $3: 00$ & $-0: 23$ \\
\hline $\begin{array}{l}\text { Esparcimiento, } \\
\text { cultura y } \\
\text { entretenimiento }\end{array}$ & $2: 07$ & $1: 46$ & $+0: 21$ & $2: 04$ & $3: 34$ & $-1: 0^{*}$ \\
\hline $\begin{array}{l}\text { Cuidados } \\
\text { personales }\end{array}$ & $1: 53$ & $2: 26$ & $-0: 33^{*}$ & $2: 10$ & $2: 38$ & $-0: 28$ \\
\hline Total & $\begin{array}{c}599 \\
(22.4)\end{array}$ & $\begin{array}{c}762 \\
(28.5)\end{array}$ & $\begin{array}{l}1361 \\
(50.9)\end{array}$ & $\begin{array}{c}464 \\
(17.4)\end{array}$ & $\begin{array}{c}847 \\
(31.7)\end{array}$ & $\begin{array}{l}1311 \\
(49.1)\end{array}$ \\
\hline
\end{tabular}




\section{Escenarios de actividad: condiciones dominantes de la actividad doméstica}

Los resultados del análisis contextual se presentan en la Tabla 4.

Tabla 4.

Escenarios de actividad urbano y rurales

$\begin{array}{ccc}\text { HiperCódigo DEL ESCENARIO PRINCIPAL UE/DÍA/DUR/ } \\ \text { CS } \\ \text { Trabajo en casa } & \text { Rural } & \text { CC_ES_M_A } \\ & \text { Urbano } & \text { R_FS_M_S } \\ & \text { Rural } & \text { R_ES_L_A }\end{array}$

Esparcimiento, cultura y entretenimiento

Urbano R_FS_M_S

Cuidados personales

Urbano

CC_ES_M_A

B_ES_C_S

Ubicación espacial (UE) - $\mathrm{R}$ = recámara, $\mathrm{B}=$ baño, $\mathrm{S}=$ sala, $\mathrm{CM}=$ comedor, $\mathrm{CC}=$ cocina

Día de la semana (DíA) - ES= entre semana, FS = fin de semana

Duración (DUR) - $C=$ corta (1 a 14 minutos), $M=$ media (15 a 29

minutos), $\mathrm{L}=$ larga (más de 15 minutos)

Contacto social (CS) - $\mathrm{S}=$ solo, $\mathrm{A}=$ acompañado

\section{Hipercódigos del trabajo en casa}

En las viviendas rurales, la actividad de trabajo en casa ocurre más frecuentemente en la cocina (CC), en los días entre semana (ES), con una duración media (M) y en compañía de otras personas ( $A=$ acompañado). El hipercódigo correspondiente es CC_ES_M_A. Por otro lado, en las casas urbanas, la actividad de trabajo en casa ocurre más frecuentemente en la recámara $(R)$, en los fines de semana $(F S)$, con una duración media $(M)$ y en ausencia de otras personas $(S=-$ solo). El hipercódigo correspondiente es R_FS_M_S.

\section{Hipercódigos del esparcimiento, cultura y entretenimiento}

En las casas rurales, la actividad de esparcimiento ocurre más frecuentemente en la recámara $(R)$, en los días entre semana (ES), con una larga duración (L) y en compañía de otras personas ( $\mathrm{A}=$ acompañado). El hipercódigo Correspondiente es R_ES_L_A.

En las casas urbanas, la actividad de esparcimiento ocurre más frecuentemente en la recámara $(R)$, en los fines de semana (FS), con una duración media (M) y en ausencia de otras personas ( $\mathrm{S}=$ solo). El hipercódigo correspondiente es R_FS_M_S.

\section{Hipercódigos de los cuidados personales}

En las casas rurales, la actividad de cuidados personales ocurre más frecuentemente en la cocina (CC), en los días entre semana (ES), con una duración media (M) y en compañía de otras personas ( $A=$ acompañado). El hipercódigo correspondiente es CC_ES_M_A.

En las casas urbanas, la actividad de cuidados personales ocurre más frecuentemente en el baño (B), en los días entre semana (ES), con una corta duración (C) y en ausencia de otras personas $(\mathrm{S}=$ solo). El hipercódigo correspondiente es B_ES_C_S.

Las actividades rurales tienen como lugar significativo la cocina. Este es un espacio primordial tanto para las actividades de trabajo como para las de cuidado personal. Estas mismas actividades son realizadas por los moradores urbanos en contextos más privados, la recámara y el baño. En las viviendas urbanas los residentes se bañan en un cuarto privado al fondo de la casa, los habitantes rurales suelen hacerlo en la cocina o en el patio. Las actividades y los espacios donde éstas ocurren sugieren que los episodios reportados por los residentes rurales están mayormente orientados a otras personas, mientras que los urbanos se encuentran dirigidos hacia la misma persona.

Tanto en la vivienda urbana como en la rural, las actividades de cuidados se presentan principalmente en los días entre semana. Para el trabajo en casa y el esparcimiento en la zona rural, el día imperante es entre semana y para la urbana en fin de semana. Las actividades de esparcimiento, para los participantes rurales, son de larga duración, mientras que las actividades urbanas de cuidados personales son de corta duración. Existen, por tanto, diferencias entre ambos ambientes en cuanto a los factores temporales de la actividad doméstica.

Las actividades en la vivienda rural ocurren principalmente en compañía de otras personas, aun cuando se trate de actividades que requieren privacidad, como las de cuidados personales. En cambio, las actividades urbanas ocurren sin la presencia de personas.

\section{Discusión}

La psicología en general, y la psicología ambiental en particular, deben atender todo el panorama de sus objetos de estudio. Sólo de esta forma se podrán comprender las relaciones entre el ambiente, en específico la casa, y el comportamiento. Las casas, en sus diversos contextos, deben forman parte de un tópico de estudio que incluya el análisis de las semejanzas y diferencias en el comportamiento. Si únicamente nos enfocamos en la vivienda 
urbana, no podremos apreciar la compleja variabilidad de las relaciones ambiente-comportamiento.

Por otro lado, la atención enfocada exclusivamente en la vivienda urbana promueve que se le otorgue una relevancia sesgada, al mismo tiempo que subestimamos la importancia de la vivienda rural. Como consecuencia, las necesidades y problemáticas de los moradores rurales son ignoradas tanto por los científicos como por los responsables de la generación de políticas públicas.

Los modelos y teorías psicológicas sobre la vivienda, igualmente, explicarán de forma parcial el comportamiento si se considera sólo la vivienda urbana. Los modelos psicológicos sobre la vivienda, como el de Habitabilidad de la Vivienda Urbana (Mercado, Ortega, Luna \& Estrada, 1995), y el estudio arquitectónico de la misma, podrán enriquecer el alcance de las explicaciones y las formas de interpretación si se incorporara el comportamiento en las casas rurales.

A pesar de que los asentamientos urbanos y rurales configuran realidades diferentes para sus habitantes, éstas también son conformadas por la profunda influencia de las necesidades inherentemente humanas. En este sentido, los estudios comparativos sobre el comportamiento en las viviendas urbanas y rurales son importantes y necesarios para definir los límites y alcances de las explicaciones, modelos y teorías, así como para orientar las soluciones y políticas públicas hacia las realidades de cada asentamiento.

La incorporación de diferentes aproximaciones a la investigación en psicología ambiental en general, y sobre la vivienda en particular, puede enriquecer y ampliar la perspectiva tanto de la concepción misma de los fenómenos como de los métodos para investigarlos. Una de las aproximaciones es el enfoque sobre el uso del tiempo (Pentland, Harvey, Lawton \& McColl, 2002). Éste es especialmente útil para el estudio de la vivienda, ya que ofrece patrones globales de comportamiento dentro del contexto residencial donde éstos se desarrollan. Por tanto, esta aproximación aporta una visón y una metodología para estudiar la complejidad de la vida diaria de las personas en sus casas, la cual involucra diferentes factores, tanto sociales, como espaciales y temporales, los cuales varían dependiendo del contexto.

Los estudios sobre uso del tiempo, junto con la técnica de diario de actividad, proveen una ventana a los estilos de vida de los residentes urbanos y rurales. A través de esta ventana es posible apreciar una visión más completa del comportamiento en la vivienda. Los resultados proveen una base rica, objetiva y replicable sobre la cual hacer juicios empíricos. El análisis contextual, por medio de los hipercódigos, genera evidencia invaluable para comprender el comportamiento de los mo- radores en general, así como las diferencias conductuales entre distintos tipos de ambientes domésticos. Esta información es valiosa como guía para la planeación y desarrollo de políticas de los asentamientos. También brinda evidencia sólida para el diseño de las viviendas orientado a la vida cotidiana de los moradores.

Los resultados de este estudio muestran que un mismo escenario conductual, la vivienda, puede contener episodios de actividad diferentes de acuerdo al tipo de asentamiento, urbano o rural, donde ésta se ubica. Estas diferencias se asocian con aspectos espaciales (la ubicación de la actividad, por ejemplo cocina, baño), sociales (la compañía) y los temporales (día entre semana o fin de semana). Las casas urbanas y rurales ofrecen diferentes oportunidades-estructuras (Saegert \& Winkel, 1990) para el desarrollo de las actividades. Los episodios de actividad predominantes, tanto voluntarios como involuntarios, pueden ser facilitados u obstaculizados por los elementos físicos y sociales del lugar, así como por la ubicación temporal.

Las casas rurales, en contraste con las urbanas, presentan mayores condiciones de pobreza, hacinamiento y falta de equipamiento. Estas condiciones promueven menos oportunidades para las actividades, por lo que éstas presentan menor diversidad que las urbanas.

Las oportunidades para lograr privacidad también se encuentran constreñidas por las características de las viviendas rurales. Jiménez y Verduzco (2009), al analizar la configuración espacial de casas urbanas y rurales, encontraron que las asentadas en el ambiente rural de su investigación, eran menos profundas que las que estudiaron en la ciudad. La profundidad de la casa ofrece mayores oportunidades para que los moradores puedan distanciarse de otras personas (Evans, Lepore \& Shroeder, 1994). Cuando existe mayor profundidad, los habitantes pueden alejarse hacia otros lugares dentro de la misma casa, y así lograr más privacidad. Por tanto, en las casas rurales, al ser menos profundas, las actividades de los participantes se desarrollaron, con mayor frecuencia, en presencia de otras personas. De forma contraria, la profundidad de las casas urbanas brinda oportunidades-estructuras para que los residentes logren distanciarse, por lo que los participantes del estudio reportaron menor número de personas presentes al realizar sus actividades.

La ubicación de la actividad también se encuentra relacionada con las oportunidades y restricciones de la casa. Los habitantes rurales reportaron mayor uso de la cocina, un espacio público, mientras que los urbanos utilizaron más la recámara, un espacio privado. Las actividades rurales, al desarrollarse en mayor medida en presencia de otras personas, requieren un espacio público. Las 
actividades urbanas, al contario, al realizarse de forma solitaria se ubican mayormente en un espacio privado.

El tiempo también posee un rol importante en las oportunidades-estructuras para el comportamiento en las casas. De acuerdo a INEGI ( 2015) la mitad de la población ocupada (50.6\%) se encuentra en las ciudades grandes, mientras el $13.9 \%$ se concentra en las zonas rurales con menos de 2500 habitantes. De esta manera, los días entre semana están mayormente dedicados a las horas de trabajo en el ambiente urbano, mientras que en el rural hay mayor disposición de tiempo para las actividades en el hogar. Estas condiciones laborales promueven que la vida entre semana y en fin de semana sean distintas entre los ambientes rurales y urbanos.

Como los resultados indicaron, la actividad en la vivienda urbana es mayor los fines de semana, cuando no se labora, específicamente respecto al trabajo en casa y al esparcimiento. La actividad de cuidado personal es más frecuente entre semana, posiblemente debido al arreglo personal requerido para salir a trabajar. En el ambiente rural, las tres categorías de actividad prevalecieron en los días entre semana. La disposición de tiempo entre semana para los habitantes rurales y en fin de semana para los urbanos, genera estas oportunidades para la actividad doméstica.

\section{Conclusiones}

Los resultados de este estudio indican que las realidades de las viviendas urbanas y rurales albergan distintas interacciones entre las formas de vida y el espacio. La psicología ambiental, por tanto, debe incorporar los asentamientos rurales en las agendas de investigación para lograr una comprensión más exhaustiva del comportamiento en la vivienda.

El enfoque del uso del tiempo, la técnica de diario de actividad y el análisis contextual, son aproximaciones útiles y convenientes para obtener un reflejo de la rutina diaria de las personas en sus casas. Este tipo de estudios permite realizar comparaciones para identificar las diferencias entre los asentamientos urbanos y rurales. Con el análisis contextual y el uso de hipercódigos se puede obtener un panorama valioso de la actividad dominante en la vivienda. A través de este análisis es posible comprender cómo los patrones de actividad en los distintos ambientes domésticos están influenciados por el contexto. Los resultados obtenidos con este análisis brindan información concentrada y global que podría orientar las políticas e intervenciones para el bienestar de los moradores.
Es importante y necesaria más investigación, la cual incluya muestras más amplias y diversas para enriquecer el panorama aquí expuesto. Además, es conveniente la incorporación de otros factores, como la satisfacción de los participantes con la actividad o con los espacios donde ésta ocurrió. Estas direcciones a seguir podrán aportar evidencia y nuevas propuestas para la investigación del comportamiento en la vivienda. Si bien en este documento se resaltó el potencial de la metodología de uso del tiempo, es importante considerar algunas limitaciones inherentes al estudio. La primera tiene que ver con la generalización de los resultados. Ya que se consideró únicamente la información sólo de 120 participantes durante dos días de la semana, por tanto, puede ponerse en cuestión lo representativo de la información.

En segundo lugar, es probable algún efecto asociado a la deseabilidad social, de tal forma que existan omisiones de algunas actividades, principalmente de aquellas que se consideran privadas. En tercer lugar, es posible que ciertas actividades, como el estar solamente meditando, sin hacer nada, o aquellas con una duración muy breve, no se hayan reportado. A pesar de las limitaciones expuestas, los estudios sobre el uso del tiempo en diversos países han reportado evidencia acerca de la confiabilidad y validez de esta metodología (Robinson, 1988).

\section{Referencias}

Aguilar, B. (2001). Estado del arte sobre la arquitectura vernácula en México. Documento presentado en 3er Seminario sobre vivienda rural y calidad de vida en los asentamientos rurales, Santiago de Cuba.

Baptista, F. J. (2001). Aproximación metodológica desde la Psicología Social a la investigación en zonas rurales. Revista Española de Estudios Agrosociales y Pesqueros, 191, 225-236.

Evans, G. W., Saegert, S. \& Harris, R. (2001). Residential density and psychological health among children in low-income families. Environment and Behavior, 33(2), 165-180.

Evans, G., Lepore, S. \& Shroeder, A. (1994). Crowding and spatial syntax. En S. J. Neary, M. S. Synes \& F. E. Brown (Eds.). The urban experience. London: E \& F. N. Spon.

Harvey, A. (2002). Guidelines for time use data collection and analysis. En W. E. Pentland, A, S. Harvey, P. Lawton \& M. A. McColl (2002). Time use research in the social sciences. Nueva York: Kluwer Academic Publishers.

Helbok, C. (2003). The practice of psychology in rural communities: Potential ethical dilemmas, Ethics \& Behavior, 13(4), 367-384.

Hicks, L. (2002). Rural health care. Recuperado de https://www.apa.org/practice/programs/rural/hicks.pdf. 
INEGI. (2005). Encuesta Nacional Sobre Uso del Tiempo 2002. Tabulados definitivos. Recuperado de http://cedoc.inmujeres.gob.mx/documentos download/100491.pdf

INEGI (2015). Resultados de la encuesta nacional de ocupación y empleo. Cifras durante el primer trimestre de 2015. Recuperado de http://www.inegi.org.mx/saladeprensa/boletines/2015/ enoe_ie/enoe_ie2015_05.pdf

Jiménez, E. O. \& Verduzco, G. (2009). La sintaxis espacial de la vida doméstica. Una comparación urbano-rural. Palapa, Revista de Investigación Científica en Arquitectura, 4(2), 45-52.

Larson, R. (1989). Beeping children and adolescents: A method for studying time use and daily experience. Journal of Youth and Adolescence, 18(6), 511-530.

Martínez, R. (1991). Métodos de investigación en psicología ambiental. En F. Jiménez y J. I. Aragonés (Eds.), Introducción a la Psicología Ambiental. Madrid: Alianza

Mercado, S., Ortega, P., Luna, G., \& Estrada, C. (1995). Habitabilidad de la vivienda urbana. México: Universidad Nacional Autónoma de México.

Michelson, W. (1990). Measuring macroenvironment and behavior: The time budget and time geography. En R. B. Bechtel, R. W. Marans y W. Michelson (Eds.), Methods in environmental and behavior research. Nueva York: Van Nostrand Reinhold.

Michelson, W. (1994). Everyday life in contextual perspective. En I. Altman y A. Churchman (Eds.), Women and the environment (Vol. 13, pp. 13-42). Nueva York: Plenum Press.
Michelson, W. \& Reed, P. (1975). The time budget. En W. Michelson (Ed.), Behavioral research methods in environmental design. Pennsylvania: Dowen, Hutchinson \& Ross.

Pentland, W. E., Harvey, A. S., Lawton, M. P. \& McColl, M. A. (Eds.). (2002). Time use research in the social sciences. Nueva York: Kluwer Academic Publishers.

Pentland, W. E., \& McColl, M. A. (2002). Application of time use research to the study of life with a disability. En W. E. Pentland, A, S. Harvey, P. Lawton y M. A. McColl (Eds.), Time use research in the social sciences. Nueva York: Kluwer Academic Publishers.

Rapoport, A. (2005). Culture, architecture, and design. Chicago: Locke Science Publishing Company.

Robinson, J. P. (1988). Time-diary evidence about the social psychology of everyday life. En J. E. McGrath (Ed.), The social psychology of time (pp. 134-148). EUA: Sage Publications.

Saegert, S. \& Winkel, G. (1990). Environmental Psychology. Annual Review of Psychology, 41, 441-477.

Sánchez, Q.C. (2009). Psicología en ambiente rural. México, D.F.: Plaza y Valdés.

United Nations. (2015). Urban and rural areas 2007. Recuperado de https://www.un.org/development/desa/es/news/ population/world-urbanization-prospects-2014.html

Wells, N. M. \& Evans, G. W. (2003). Nearby nature: A buffer of life stress among rural children. Environment and Behavior, 35(3), 311-330.

Werner, C. M., Altman, I., \& Oxley, D. (1985). Temporal aspects of homes: A transactional perspective. In I. Altman y C. M. Werner (Eds.), Home environments: Human behavior and the environment (Vol. 8, pp. 1-32). New York: Plenum. Published,

Wicker, A. (1979). An introduction to ecological psychology. Editorial CA: Brooks/Cole, Monterrey. 
Artículo Empiríco | Uso del espacio doméstico. Análisis comparativo urbano/rural... I Jiménez-Rosas \&Z Negrete-Rodríguez

\section{Meta-Análisis del Artículo}

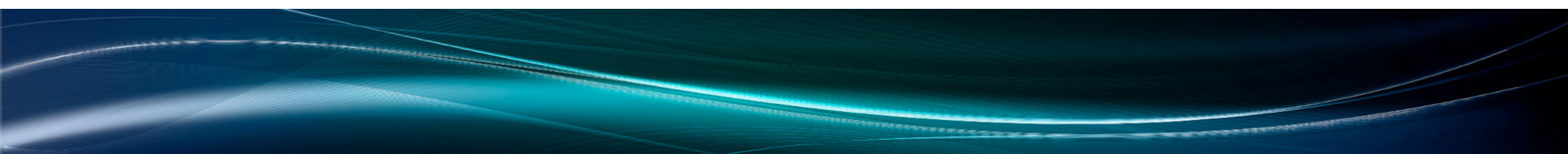


Revista Digital Internacional de Psicología y Ciencia Social |Vol. 2 | Núm. $1 \mid$ Enero-Junio 2016 | ISSSN 1405-5802

\section{Dimensión Cuantitativa}

\section{Perfil de Evaluación entre pares}
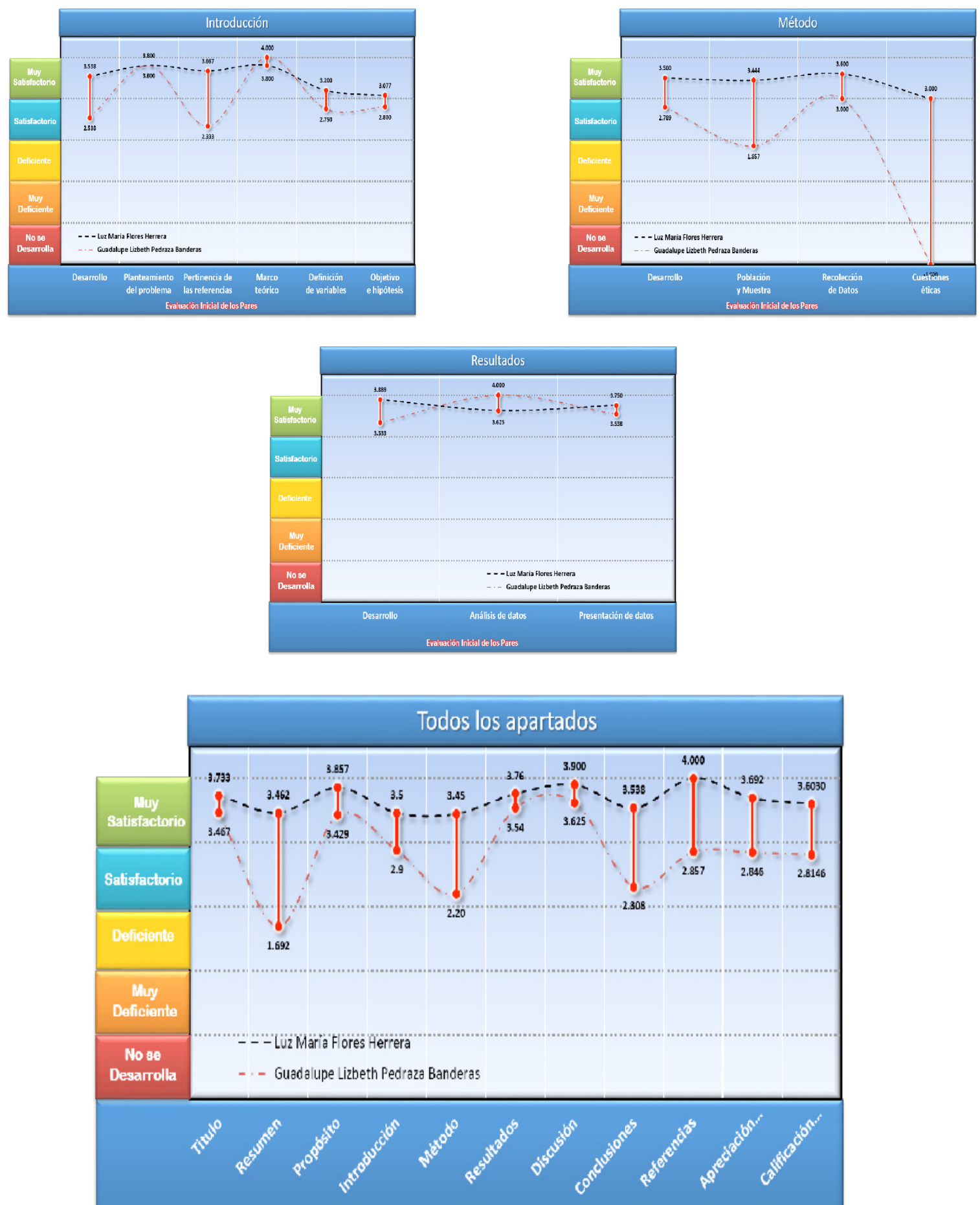

Evaluación Inicial de lo5 Pares 


\section{Índice de Concordancia}

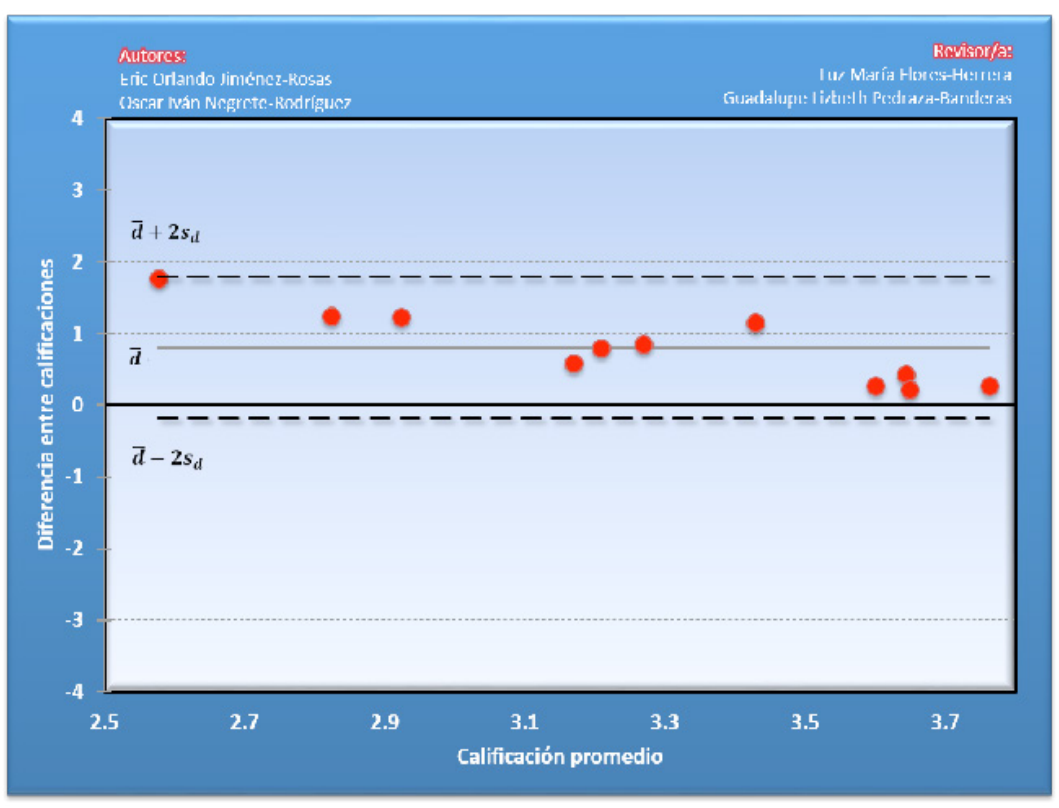

Índice de Acuerdo
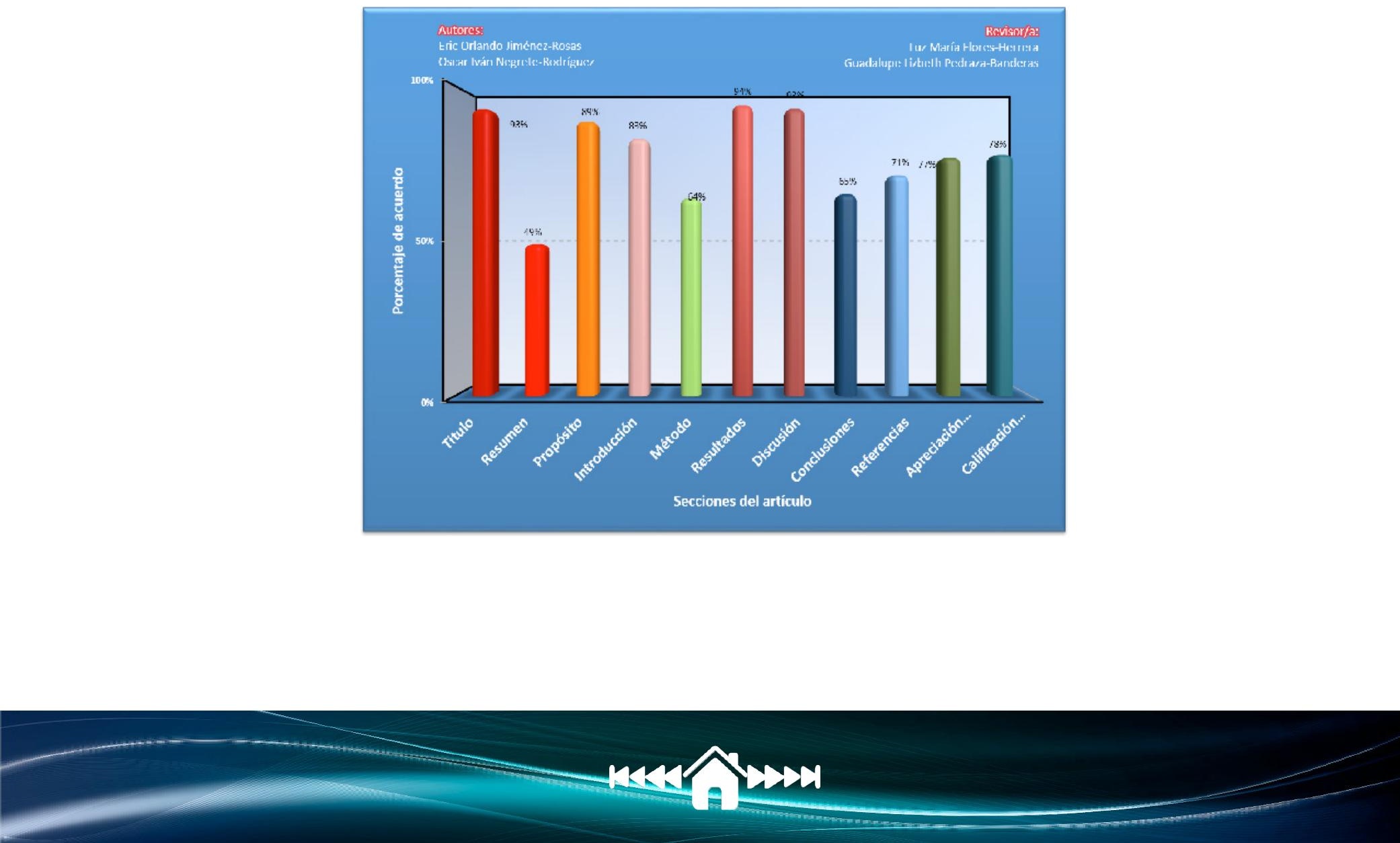


\begin{tabular}{|c|c|}
\hline Revisor 1 & Revisor 2 \\
\hline Luz María Flores-Herrera & Guadalupe Lizbeth Pedraza-Banderas \\
\hline \multicolumn{2}{|c|}{ Título/Autoría } \\
\hline El título es correcto, sin sugerencias de mejora. & Omitir el artículo “un” \\
\hline \multicolumn{2}{|c|}{ Resumen } \\
\hline $\begin{array}{l}\text { Incluir la frecuencia de las actividades rurales y urba- } \\
\text { nas según el contacto social, o bien el valor de "t" repor- } \\
\text { tando la diferencia significativa }\end{array}$ & $\begin{array}{l}\text { No contiene el objetivo, presenta fallas de uso de sig- } \\
\text { nos ortográficos, en particular, las comas, además el } \\
\text { uso del término éstas es confuso, pues se reduce a las } \\
\text { actividades, aunque propiamente se está haciendo a las } \\
\text { personas. }\end{array}$ \\
\hline \multicolumn{2}{|c|}{ Próposito del Estudio } \\
\hline $\begin{array}{l}\text { El propósito es claro y rescata la comparación entre las } \\
\text { viviendas }\end{array}$ & Sin comentarios. \\
\hline \multicolumn{2}{|c|}{ Introducción } \\
\hline $\begin{array}{l}\text { El artículo es una evaluación y por tanto es innecesaria } \\
\text { una hipótesis, los vínculos señalados son adecuados. }\end{array}$ & $\begin{array}{l}\text { Revisar sección de redacción, citar diferentes argumen- } \\
\text { tos, así como revisar las coclusiones y discusiones, hay } \\
\text { información traslapada entre dichas secciones. }\end{array}$ \\
\hline \multicolumn{2}{|c|}{ Método } \\
\hline $\begin{array}{l}\text { En general es un buen artículo, en la evaluación anterior } \\
\text { se solicitan aspectos que no se manejan porque el estu- } \\
\text { dio es de carácter evaluativo con metodología obser- } \\
\text { vacional, de ahí que algunos conceptos como validez o } \\
\text { confiabilidad de los instrumentos no indiquen caracte- } \\
\text { rísticas psicométricas. Con respecto a los participantes, } \\
\text { si existen algunas irregularidades con la muestra, es un } \\
\text { aspecto relevante que puede quedar como sugerencia } \\
\text { para siguientes estudios. }\end{array}$ & Algunas sugerencias para hacer más clara la redacción \\
\hline \multicolumn{2}{|c|}{ Resultados } \\
\hline
\end{tabular}




\begin{tabular}{|c|c|}
\hline Revisor 1 & Revisor 2 \\
\hline $\begin{array}{l}\text { En general el análisis estadístico es correcto, existen al- } \\
\text { gunas dudas sobre todo en la DE. Con respecto a gráf- } \\
\text { cas no fueron utilizadas, y las tablas fueron adecuadas. }\end{array}$ & $\begin{array}{l}\text { En el archivo que descargue falta la tabla 1, hay algunos } \\
\text { párrafos donde la información es repetitiva y errores } \\
\text { del tiempo de redacción. }\end{array}$ \\
\hline \multicolumn{2}{|c|}{ Discusión } \\
\hline $\begin{array}{l}\text { La discusión rescata los elementos teóricos y concep- } \\
\text { tuales, en las limitantes sería conveniente analizar el } \\
\text { nivel socioeconómico de los participantes, éste puede } \\
\text { ser un factor covariante para algunos de los hallazgos } \\
\text { reportadas únicamente por la V.I }\end{array}$ & $\begin{array}{l}\text { Es importante una lectura de esta sección, a mi juicio, } \\
\text { colocaron en esa parte información que va en la intro- } \\
\text { ducción, antecedentes. }\end{array}$ \\
\hline \multicolumn{2}{|c|}{ Conclusiones } \\
\hline $\begin{array}{l}\text { El artículo no señala el nivel socioeconómico de los } \\
\text { participantes, se manifiestan conclusiones de diferen- } \\
\text { cia entre las muestras (rural y urbana) sin considerar } \\
\text { posible influencia del nivel económico. En condiciones } \\
\text { urbanas se trabajo con casas construidas mientras que } \\
\text { en rurales con autoconstruidas. }\end{array}$ & Sin comentarios \\
\hline \multicolumn{2}{|c|}{ Referencias } \\
\hline $\begin{array}{l}\text { Es una sección correcta acorde con los lineamientos del } \\
\text { APA. }\end{array}$ & $\begin{array}{l}\text { Me parecen pocas referencias, sobre todo en lo relati- } \\
\text { vo al modelo que abordan. Apegándose al manual APA } \\
\text { existen cambios recientes que no se consideraron, por } \\
\text { ejemplo no emplear la letra "y", sino el signo \&. }\end{array}$ \\
\hline
\end{tabular}


Revista Digital Internacional de Psicología y Ciencia Social |Vol. 2 | Núm. $1 \mid$ Enero-Junio 2016 | ISSSN 1405-5802

Historia del Proceso

\section{EDITORIAL}

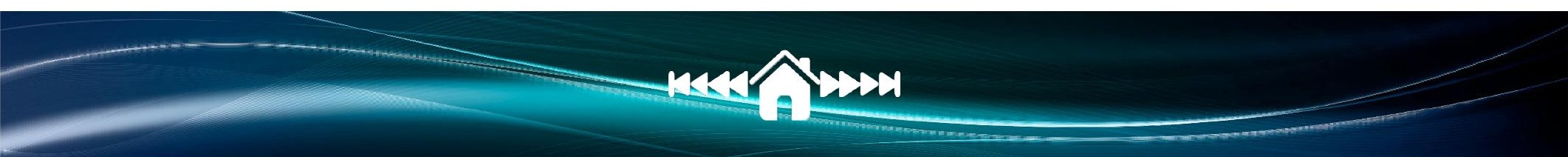

